\title{
CIÒ CHE CI SPEZZA IL CUORE E CI TRASFORMA IN MISSIONARIE: NELLE VIE DELLA SPERANZA INSIEME AI MIGRANTI
}

\author{
Terezinha Lúcia Santin, Ivanir Ana Filipi, Gjeline Preçi
}

Questo scritto ha lo scopo di presentare la nostra esperienza missionaria immersa nella realtà migratoria della Diocesi di Siracusa. Non abbiamo intenzione di fare un'analisi approfondita dei numeri e della realtà migratoria, ma una riflessione illustrativa e comunicativa di un processo recente, cioè di 15 mesi di presenza missionaria nella diocesi. Quindi ci limitiamo a descrivere quello che osserviamo della realtà migratoria locale, dell'azione di governo, della società e della Chiesa nella società e la nostra esperienza ancora in embrione in questa missione. Includiamo alcuni racconti di migranti, che illustrano la loro situazione di vita, e alcune riflessioni sulle sfide pastorali.

Da secoli le popolazioni del mondo decidono, per propria volontà $\mathrm{o}$ forzatamente, di lasciare la propria terra per spostarsi in altri paesi alla ricerca di migliori condizioni di vita. Questa realtà di migrazione forzata, a causa di guerre e persecuzioni, costringe, sempre di più, uomini e donne affamati, feriti e sfruttati, a fuggire dal proprio paese. II Rapporto Global Trends 2014 dell'UNHCR mostra che negli ultimi cinque anni sono scoppiate almeno 15 nuove guerre: otto in Africa (Costa d'Avorio, Repubblica Centrafricana, Libia, Mali, nord-est della Nigeria, Repubblica Democratica del Congo, Sud Sudan e Burundi), tre in Medio Oriente (Siria, Iraq e Yemen), una in Europa (Ucraina) e tre in Asia.

Le cause economiche, politiche, religiose e culturali, che producono le migrazioni, impediscono di precisare il numero di migranti e profughi che sono giunti in Europa in quest'ultimo tempo. Gli sbarchi dei profughi in fuga dalle guerre continuano incessantemente, dalla Siria, dall'Eritrea, dalla Somalia, dall'Egitto, dell'Iraq, dall'Afghanistan, dal Sudan e da altri paesi distrutti da guerre e persecuzioni. Esseri umani scappano dalla fame e dalla disperazione dei loro

1 Suore Missionarie Scalabriniane, Comunità di Siracusa. Siracusa, Italia. 
paesi senza futuro. Arrivano uomini, donne, bambini non accompagnati, intere famiglie. Arrivano le salme, su quei barconi maledetti. La Guardia Costiera sbarca nel porto di Augusta con alcuni dei tanti corpi che non sono riusciti a vincere le tribolazioni dell'attraversata del mare.

Come conseguenza della sua posizione geografica I'Italia, negli ultimi anni, è stata sempre più coinvolta in queste dinamiche migratorie, specificamente nell'accoglienza dei migranti che arrivano via mare. La Sicilia, in modo particolare, è il crocevia di migranti provenienti dall'Asia e dall'Africa, che sbarcano nei porti, soprattutto a Palermo, Catania, Messina, Pozzalo, Trapani, Agrigento, Augusta.

Nei racconti delle sofferenze dei migranti possiamo capire in che condizioni arrivano in questa regione:

Non ricordo bene cosa era successo quel giorno con l'aereo sopra di noi e in quel momento tutti scappavano per salvarsi. Ricordo solo tantissimi morti, nelle strade sono stati uccisi a colpi di pistola, erano uomini del governo. Non voglio più tornare in Siria perché ci sono tanti problemi con tutto quello che succede in Siria.

(Ragazzo accolto nel centro di prima accoglienza Umberto I; non si identifica per paura).

Sono tanti i problemi durante il viaggio nel mare. Durante il mio viaggio c'è stato temporale e mare mosso, è stato terribile, ho creduto di morire, mi sembrava impossibile arrivare viva, è assurdo. II viaggio è durato 5 giorni. $\mathrm{Al}$ buio in mezzo al mare con la barca, sono partita dalla Libia e per partire ho dovuto pagare 700 dollari.

(Ragazza eritrea che ci ha raccontato il suo viaggio verso Siracusa).

Sono stata 6 mesi in Libia ho visto molte cose strane, in carcere sono detenuti quasi tutti uomini, tanti eritrei e somali. Li picchiavano tutti i giorni. Sono stata 6 mesi in carcere in Libia, ogni giorno la polizia mi faceva del male, ogni giorno, non potevo fare nulla (...). La situazione in Libia è davvero incredibile. (Giovane donna che ci ha raccontato le sofferenze in Libia).

Arrivano molti adolescenti abbandonati a se stessi. Sono minori che rischiano la propria vita, come purtroppo attestano i naufragi che si sono verificati al largo delle coste di Lampedusa, del Salento e in tanti altri luoghi. Ma è l'intero viaggio, non solo quello in mare, a esporli a gravi rischi. I minori stranieri non accompagnati che arrivano in Italia hanno infatti alle spalle tragitti che talvolta sono durati anni, durante i quali hanno vissuto esperienze drammatiche. Per illustrare facciamo riferimento a un caso che ancora oggi seguiamo. Circa 8 mesi fa una ragazza appena sbarcata nel porto di Augusta è stata portata al reparto psichiatrico dell'ospedale Umberto I di Siracusa. A poco a poco abbiamo capito il suo dramma. Lei ha sofferto tanta violenza al punto da perdere l'equilibrio psichico ed emotivo. Era fuori di sé. Piccola, meno di 15 anni. Attualmente 
questa minorenne vive in una comunità di ragazze richiedenti asilo, ancora sotto cure mediche. Al solo pensare ai loro racconti le lacrime sgorgano ai nostri occhi. È incredibile!

L'organizzazione e le strutture governative per l'accoglienza dei migranti e profughi sono diverse e con scopi diversi. Ci sono anche strutture private, con risorse pubbliche, che si moltiplicano nel territorio.

I centri di prima accoglienza o di accoglienza emergenziale - CARA - sono distribuiti sul territorio nazionale a seguito di sbarchi o situazioni di emergenza. Quelli di Siracusa, di Città Giardino, di Priolo, che conosciamo noi, sono luoghi di passaggio per richiedenti asilo o profughi e minorenni. Essi arrivano, sono ospitati e rimangono lì fino a che vengono trasferiti in altri centri. La gestione di questi Centri dipende dalle prefetture. Normalmente il periodo di permanenza è corto, in modo da assicurare un rapido turnover dei richiedenti asilo i quali, in ogni caso, una volta presentata la domanda di protezione, potranno essere trasferiti nei centri di seconda accoglienza, vale a dire nelle strutture della rete SPRAR.

La rete SPRAR è costituita da Centri di piccole dimensioni per accoglienza e inserimento dei richiedenti asilo segnalati al servizio centrale da enti locali, associazioni, prefetture e questure. SPRAR è il sistema ordinario di seconda accoglienza dei richiedenti asilo e dei rifugiati.

Negli ultimi anni, a partire dal 2011, i rilevanti flussi di profughi arrivati via mare hanno congestionato il sistema di accoglienza esistente (CARA+SPRAR), obbligando il Ministero a reperire strutture di accoglienza emergenziali e "temporanee". Convenzionalmente, queste strutture vengono indicate con I'acronimo CAS (Centri di Accoglienza Straordinari).

C'è una moltiplicazione delle comunità alloggio di associazioni di accoglienza dei minori non accompagnati e famiglie. Queste comunità di seconda accoglienza vengono gestite da privati con risorse pubbliche con gli stessi criteri delle strutture governative. Anche qualche Istituto Religioso fa questa accoglienza. Molti dei minori stranieri non accompagnati sono stati accolti, e a volte "posteggiati" per mesi, in centri di prima accoglienza e successivamente hanno fatto ingresso in strutture di secondo livello, comunità alloggio, dove, anche se diciassettenni, hanno intrapreso percorsi individualizzati d'integrazione. La scuola, la formazione professionale, i tirocini formativi, quest'ultimi presso attività commerciali attente e sensibili, sono gli elementi costitutivi di progetti educativi che hanno permesso al Tribunale per i minorenni, attraverso audizioni e valutazioni individuali, di riconoscere al singolo minore, prossimo alla maggiore età, l'applicazione dell'art. 25, cioè il diritto di poter permanere nella struttura sino al completamento del progetto formativo in corso.

La Diocesi di Siracusa, soprattutto dopo la tragedia del 3 ottobre 2013 e la morte al largo di Lampedusa di 366 migranti e l'arrivo continuo di migranti 
nel porto di Augusta, ha affrontato l'emergenza con tutto il cuore e la capacità ecclesiale. Dobbiamo sottolineare soprattutto l'impegno delle parrocchie di Augusta. Ad esempio, squadre di volontari presenti agli sbarchi hanno prestato servizi ai migranti; sono stati allestiti centri di accoglienza; persone legate ad associazioni fanno un servizio di supporto e assistenza ai minori migranti. La Chiesa locale, attraverso, soprattutto, le parrocchie, I'Ufficio Migrantes, la Caritas e i cristiani sensibili al dramma dei migranti, è attenta e aperta alle esigenze di emergenza dei migranti. In questo contesto di collaborazione si inserisce la presenza di noi suore scalabriniane.

L'obiettivo primario della nostra presenza è quello dell'aiuto e dell'amore fraterno verso i migranti, con percorsi di collaborazione con la comunità ecclesiale locale e le forze sociali. II nostro servizio è iniziato con la conoscenza della realtà locale ed ecclesiale. Abbiamo preso contatto con le strutture governative, con le parrocchie più sensibili e le associazioni che lavorano per i migranti.

Nei primi dieci mesi di presenza in questa diocesi abbiamo avuto l'opportunità di partecipare all'accoglienza dei migranti nel porto di Augusta, insieme alla Caritas e altri volontari. Attualmente, con i cambiamenti politici e strategici del governo italiano, la nostra presenza nel porto viene limitata. Così anche la Chiesa locale ha difficoltà ad essere più attiva e propositiva nel servizio ai migranti. Però la missione non si ferma. La nostra collaborazione si realizza oggi soprattutto nella prima accoglienza dei tanti profughi che approdano alle coste siracusane e di Augusta, dove arrivano privi di tutto, solo con quello che hanno addosso, oppure con quello che sono riusciti a salvare dalle acque, che non è molto. Le principali attività che svolgiamo a Siracusa sono le visite agli ospedali per coloro che arrivano malati o feriti, azioni di supporto per l'elaborazione dei traumi vissuti, piccole assistenze sanitarie, orientamenti per il proseguo del cammino e, soprattutto, ascolto, ascolto, ascolto. Incontriamo i migranti anche all'ingresso dei centri di prima accoglienza, nei luoghi ecclesiali in cui si riuniscono e lungo le vie della città. Consegniamo loro vestiti e scarpe, prelevate dal nostro magazzino, riservato a questo scopo, aiutandoli così alle prime necessità. Questo gesto ci permette di avere altri incontri in locali favorevoli per l'ascolto e la presenza fraterna.

Un altro impegno che abbiamo è quello di approfondire la consapevolezza e la qualifica di coloro che agiscono nell'accoglienza e nella pastorale dei migranti e rifugiati, attraverso la formazione interculturale. Per questo, insieme all'Ufficio per la Pastorale della Cultura e in collaborazione con la Caritas Diocesana, I’Ufficio Migrantes e i Volontari per le migrazioni stiamo effettuando una serie di incontri sul tema della cultura dell'accoglienza nella dimensione ecclesiale, con attenzione anche alle cause dell'immigrazione. 
Comunque le sfide che la realtà attuale pone alla Chiesa sono tante. Quelle che, a nostro giudizio, emergono con più forza sono: cercare di stabilire delle relazioni con i migranti e verificare quale aiuto la comunità cristiana può offrire loro nelle problematiche che li coinvolgono. Ad esempio capire cosa pensano o che progetti hanno i minori migranti che raggiungono la maggiore età (i diciotto anni) e non possono più contare sui fondi del Ministero dell'Interno destinati ai minori non accompagnati e consequentemente devono lasciare le comunità alloggio. Altra sfida è quella di capire i motivi per cui le autorità non ci lasciano arrivare al porto di Augusta al momento degli sbarchi. Come trovare la via giusta per essere presenti nell'accoglienza nel porto? Capire l'incidenza delle nuove norme sulla vita dei migranti, dallo sbarco all'hotspot, all'espulsione. Le violazioni dei diritti, come distinzioni arbitrarie tra migranti economici e richiedenti protezione internazionale, espulsioni lampo, metodi ingannevoli per ottenere le impronte, carenza di informazioni sui loro diritti e il loro destino. Un'altra sfida è quella di sistematizzare l'esperienza fatta finora e tracciare un programma missionario con le nostre caratteristiche, sottolineando sempre il lavoro in linea con la diocesi e in comunione e partecipazione pastorale.

Insomma, il nostro impegno è quello di fare di tutto affinché i migranti possano trovare un'accoglienza dignitosa che dia loro il coraggio e, soprattutto, la speranza che Dio non li ha abbandonati durante questo cammino arduo e difficile. Tutto ciò per noi diventa chiaro quando ascoltiamo le espressioni del loro cuore: "oggi voi avete fatto per noi come Gesù, ci ha lavato, non solo i piedi ma tutto il nostro corpo"; "voi siete la nostra famiglia"; "sono grande ma per me voi siete la mamma e io il bambino"; "grazie mamma, grazie..."; "mamma ti chiamo perché mi fa male la pancia, non riesco più a mangiare la pasta...". Queste e molte altre espressioni confermano la nostra missione in questa realtà. 